\title{
Work-Focused Cognitive Behavioral Therapy to Complement Vocational Services for People With Mental IIIness: Pilot Study Outcomes Across a 6-Month Posttreatment Follow-Up
}

\author{
By: Marina Kukla \\ HSR\&D Center for Health Information and Communication, Richard L. Roudebush Veterans Affairs Medical Center, \\ Indianapolis, Indiana, and Department of Psychology, Indiana University-Purdue University Indianapolis; \\ Michelle P. Salyers \\ Department of Psychology, Indiana University-Purdue University Indianapolis, and ACT Center of Indiana, Indianapolis, \\ Indiana
}

Amy M. Strasburger

Richard L. Roudebush Veterans Affairs Medical Center, Indianapolis, Indiana

Annalee Johnson-Kwochka

Department of Psychology, Indiana University-Purdue University Indianapolis

Emily Amador

Marian University College of Osteopathic Medicine

Paul H. Lysaker

Richard L. Roudebush Veterans Affairs Medical Center, Indianapolis, Indiana, and Department of Psychiatry, Indiana University School of Medicine

Acknowledgement: This study was supported by Veterans Health Administration Rehabilitation Research and Development Grant D0943-W.

Note: Sandra G. Resnick served as the action editor for this journal._SGR

\section{Abstract:}

Objective: People with mental illness frequently have trouble obtaining and keeping competitive employment and struggle with on-the-job performance. To address these issues, the manualized, group-based, 12-session Cognitive Behavioral Therapy for Work Success (CBTw) intervention was developed and tested in an open trial. Although posttreatment work outcomes were promising, lasting effects associated with the intervention are unknown.

Method: This article presents the 6-month posttreatment work outcomes of the open trial of CBTw in 52 adults with mental illness who were concurrently receiving VA vocational services. Work outcomes included work status, hours worked and wages earned, steady work status, and work performance and effectiveness. Data were analyzed using repeated measures analysis of variances (ANOVAs) and within groups t tests. Results: Findings demonstrate that $75 \%$ of unemployed participants at baseline obtained competitive work during the study period. During the 6-month follow-up period, $73 \%$ of workers attained steady work status (i.e., working at least $50 \%$ of the follow-up period). In addition, during the 6 -month follow-up period, working participants averaged significantly more hours of work per week and higher wages earned per hour as compared with the baseline period and the 12-week postintervention period. Finally, among workers, reports of work effectiveness remained high at 6 months and work productivity disruptions remained low at follow-up.

Conclusions and Implications for Practice: These findings suggest that CBTw may potentially be a useful tool to enhance the effects of vocational programs. Future work should test CBTw in a randomized controlled trial and examine strategies for implementation in real-world vocational service settings.

This work is written by a US Government employee and is in the public domain in the US. https://doi.org/10.1037/prj0000365 
People with mental illness have well documented difficulties obtaining competitive work in the community (e.g., Pogoda et al., 2016; Zivin et al., 2011). Those who do obtain employment frequently experience disruptions in work effectiveness and productivity (Adler et al., 2011), and are at a higher risk for employment loss (BurnettZeigler et al., 2013); indeed, many people lose their jobs within a matter of months (Bond \& Kukla, 2011). Quality vocational services exist to assist these individuals in achieving their vocational goals, however, a sizable portion continue to struggle (Abraham, Yosef, Resnick, \& Zivin, 2017). In response, complementary psychosocial interventions have begun to be developed to ameliorate many commonly identified personal work barriers, such as low work-related self-efficacy (Zivin et al., 2016), poor sense of self as a productive worker (Williams, Fossey, Corbière, Paluch, \& Harvey, 2016), low self-esteem (Corbière, Lanctôt, Sanquirgo, \& Lecomte, 2009), and lack of work-specific coping skills (Kukla, Bonfils, \& Salyers, 2015; Kukla, McGuire, \& Salyers, 2016). Recently, we published a paper reporting posttreatment outcomes of an open trial examining the Cognitive Behavioral Therapy for Work Success (CBTw) intervention (Kukla, Strasburger, \& Lysaker, 2016; Kukla, Strasburger, Salyers, Rollins, \& Lysaker, 2018). CBTw is designed to augment existing individualized vocational services and is particularly geared toward persons with mental illness have persistent struggles with work functioning. A previous study exploring participant perspectives on CBTw suggested potential pathways or targets by which the intervention may effect change, including cognitive restructuring, behavioral coping strategies, problem-solving for work barriers, meaningful reflection on oneself as a worker, and instilling hope that succeeding in work is possible (Kukla, Strasburger, Salyers, Rattray, \& Lysaker, 2017).

The CBTw open pilot demonstrated that half of unemployed participants at baseline acquired competitive work during the follow-up period and, compared with the 12 weeks preceding baseline, study participants worked significantly more weeks and earned significantly higher wages across the 12-week intervention period. In addition, employed participants reported a strong degree of work productivity after engaging in CBTw (Kukla et al., 2018). An outstanding question is whether improved work outcomes persist beyond the intervention period. One possibility is that participants who had yet to gain employment at posttreatment would practice and use the skills emphasized in CBTw and obtain work later. Similarly, for those who were initially successful, it is unknown whether they would continue to work and earn greater wages, achieving steady work status, or whether they would fall into the common pattern of inconsistent work and job loss across time (e.g., Bond \& Kukla, 2011). Furthermore, the trajectory of on-the-job functioning is also unclear. Specifically, as various challenges related to managing one's health and balancing work responsibilities emerge over time in competitive jobs, it is important to examine the trends of work effectiveness and work performance.

There are several reasons to anticipate that CBTw may lead to lasting improvements in work functioning. CBTw teaches techniques to challenge and adjust maladaptive patterns of thought related to work, resulting in more healthy thinking, as well as adaptive coping strategies that are well suited for everyday work settings. In addition, narrative components of the intervention are designed to help participants' grow their sense of self as a capable and productive worker. Lastly, CBTw features work success plans that offer a personalized and proactive approach that involves planning and implementing relevant strategies most important to the work success of each individual (e.g., utilizing effective communication strategies with work supervisors; attending treatment sessions to maintain sobriety; utilizing specific behavioral coping techniques to control anger at work). Given these mechanisms, it is expected that CBTw may help people with serious mental illness (SMI) sustain lasting gains in employment success. Hence, the current study sought to address these questions by examining CBTw outcomes across a 6-month follow-up period after the end of the intervention. It was hypothesized that participants would average more hours of work per week and higher hourly wages at the 6-month postfollow-up compared with the 12-week follow-up. It was also expected that at least half of the participants would become steady workers over the 6-month posttreatment follow up period, defined as working at least $50 \%$ of a given time frame. Finally, it was expected that work effectiveness would remain high and participants would report low levels of work productivity disruption at 6 months posttreatment.

\section{Method}




\section{Overview}

Fifty-two participants completed informed consent and participated in the 12-week CBTw intervention. A longitudinal open trial design was used in which competitive work outcomes were measured (a) at baseline, assessed as the period including the 12 weeks preceding CBTw enrollment, (b) at posttreatment, that is, the conclusion of the 12-week CBTw intervention period, and (c) at 6-months posttreatment (i.e., 9 months afterbaseline). The third time period was the primary target of this current study. Fifty-one veterans (98.1\%) completed the 6-month follow-up assessment.

\section{Sample}

Participants were 52 adult Veterans with SMI receiving vocational services at an urban VA medical center. Vocational services were based on Individual Placement and Support supported employment targeting competitive jobs; these services are described elsewhere (Kukla et al., 2018). Vocational services were not altered (no deviation from service model) and, thus, did not include reinforcement or presentation of CBTw content. Participants continued to receive these vocational services during the 6-month posttreatment follow up period. Study inclusion criteria were: a diagnosis of severe mental illness confirmed by medical record review, current participation in vocational services, and a competitive employment goal—regardless of employment status at the time of the study (44 were unemployed). Exclusion criteria included a significant cognitive deficit or major medical condition that would preclude participation in CBTw and/or participation in competitive work. Participants with complete follow-up assessments at 6 -months were included in the analyses $(N=51)$.

\section{Procedure}

Participants meeting inclusion criteria and participating in VA vocational services were recruited in three segments in December 2014, April 2015, and August 2015, respectively. Potential study participants completed a prescreening for eligibility, written informed consent, and a baseline assessment of background information, work, psychosocial variables, and symptoms. Participants then began a 12-week CBTw group facilitated by a licensed clinical psychologist and trained masters level clinicians. Participants were assessed at posttreatment and 6 months later after the conclusion of the intervention period. Participants were paid $\$ 30$ for completion of each assessment. Study procedures were approved by the Indiana University-Purdue University Indianapolis and Richard L. Roudebush VA Medical Center regulatory review boards.

\section{Intervention}

The CBTw program is a 12 session, manualized, group-based CBT intervention for adults with mental illness who have competitive work goals (e.g., to obtain competitive employment, or improve performance if already working). All content is tailored for competitive work in the community and is based on a cognitive and behavioral framework that focuses on increasing healthy thinking about work, managing anxiety and difficult emotions at work, bolstering work-specific coping and problem-solving, and increasing fruitful interpersonal interactions in the employment setting. In addition, the intervention includes an emphasis on managing and maintaining work success. Lastly, CBTw incorporates a narrative component designed to enhance one's sense of self as a capable and productive worker. A full description of the development and content of the intervention is provided elsewhere (Kukla et al., 2018).

\section{Measures \\ Work outcomes}

Competitive work outcomes at 6 months were employment status (unemployed/employed); competitive job acquisition; steady worker status (i.e., worked at least $50 \%$ of the follow up period); mean hours worked per week, and mean wages earned hourly in competitive jobs. Work history, measured in weeks, was defined as the longest tenure in a competitive job before the study. Work outcomes were provided via participant self-report and verified 
with VA vocational providers.

\section{Work effectiveness and productivity}

Work effectiveness and productivity among employed participants were measured using the Work and Health Interview (Stewart, Ricci, Chee, Hahn, and Morganstein, 2003). Work effectiveness was assessed by thefollowing question, "On days that you worked during the past month, how effective were you in your job?" The item was rated on a scale from $0 \%$ effective to $100 \%$ effective. Work productivity was measured using five Likert scale items assessing the degree to which productivity was diminished on days worked during the past 2 weeks, for example, "During the past 2 weeks, how often did you lose concentration at work?" The items are answered on a scale ranging from $0=$ none of the time to $4=$ all of the time. Work productivity items were then converted to quarter percentages $(0,25,50,75$, and $100 \%)$ using the approach described in Stewart et al. (2003). For example, a score of two "half of the time" corresponds to $50 \%$ reduction in work productivity in that item domain. The general work productivity score is a mean of the five item scores. Higher scores indicate greater disruption in work productivity. This Work and Health Interview has good psychometric properties in adults with mental health disorders, such as major depressive disorder (Stewart et al., 2003).

\section{Psychiatric diagnosis and symptoms}

Psychiatric diagnosis was confirmed by electronic medical record chart review. Psychiatric symptoms assessed included depressive symptoms measured by the Beck Depression Inventory-II (BDI-II; Beck, Steer, \& Brown, 1996), anxiety symptoms measured by the Beck Anxiety Inventory-2 (BAl; Beck \& Steer, 1990), and psychotic symptoms measured by the Positive and Negative Syndrome Scale (PANSS; Kay, Fiszbein, \& Opler, 1987). The $\mathrm{BDI}-\mathrm{II}$ and $\mathrm{BAI}$ are each 21-item measures scored on a 5-item Likert scale with strong reliability and validity across many studies of adults with mental illness. The PANSS is clinician-rated and consists of a total score which is the sum of all 30 items, as well as five subscales: positive, negative, hostility, cognitive, and depressive symptoms (Bell, Lysaker, Beam-Goulet, Milstein, \& Lindenmayer, 1994). The PANSS has been used and studied widely with strong psychometric properties in adults with psychosis.

\section{Analyses}

Data were analyzed utilizing SPSS 24. Descriptive statistics characterized work outcomes and adherence to statistical assumptions. Relationships between symptoms, psychiatric diagnosis, and work outcomes were examined using independent groups $t$ tests and Pearson's correlations. Repeated measures analysis of variance examined changes in continuous work outcomes between baseline, 12-week posttreatment follow-up and the 6month follow-up. Individual differences in work outcomes between time points were examined using within-groups $t$ tests. The magnitude of significant differences was characterized using Cohen's $d$ effect sizes. $p$ values were set at .05 for tests of significance.

\section{Results}

\section{Background Characteristics}

Complete background characteristics are published elsewhere (Kukla et al., 2018). Most participants were male and approximately half were African American and half White. The mean age was around 50 years, but included participants ranging from their 20s to 60s. Most participants had either a mood disorder (40\%), including bipolar disorder or major depressive disorder, or a schizophrenia-spectrum disorder (38.5\%). A minority of participants had posttraumatic stress disorder (8\%) or an anxiety disorder (13.5\%). All participants had completed high school or equivalent and the majority had at least some college. Nearly half of participants were currently receiving some assistance with housing. The mean length of time participants had been receiving vocational services at baseline was over 1 year. There were no significant relationships between work outcomes during the 6-month follow-up 
period and background characteristics, work history, psychiatric history, mental health diagnosis, or baseline symptom severity, $p>.05$ (Kukla et al., 2018).

\section{Job Acquisitions and Employment Rate}

Frequencies pertaining to employment status and job acquisition at each assessment point are presented in Table 1. During the 6-month posttreatment follow-up period, 37 participants out of the $51(72.5 \%)$ with follow updata (missing, $N=1$ ) worked in competitive jobs. Among those who were unemployed at baseline and did not work during the initial 12-week intervention period $(N=22), 11(50 \%)$ participants acquired new competitive jobs during the 6-month posttreatment follow-up period. The total job acquisition rate across the full study period among unemployed participants at baseline was $75.0 \%$ ( $N=33$ out of 44 gained competitive employment). Overall, the competitive employment rate among all participants $(N=52)$ across the study period was $78.8 \%(N=$ $41)$. Eleven participants $(22.2 \%)$ did not work at any point during the study.

\begin{tabular}{|c|c|c|c|}
\hline $\begin{array}{c}\text { Competitive } \\
\text { work }\end{array}$ & Baseline & $\begin{array}{l}12 \text { weeks } \\
\text { follow-up }\end{array}$ & $\begin{array}{l}6 \text { months posttreatment } \\
\text { follow-up }{ }^{\mathrm{a}}\end{array}$ \\
\hline Total employed & 8 & 30 & 37 \\
\hline Total unemployed & 44 & 22 & 14 \\
\hline First acquired job & - & 22 & 11 \\
\hline
\end{tabular}

${ }^{\text {a }}$ Missing, $N=1$ at 6 month posttreatment follow-up.

Frequencies of Competitive Work Status and New Job Acquisition by Study Assessment Period, $N=52$

\section{Work Descriptives Among Workers}

Among the employed group at baseline $(N=8)$, participants worked a mean of 25.1 week $(S D=2.5)$ during the 6month posttreatment follow-up period. All workers at baseline worked competitively during both follow-up periods. Among new workers who first obtained a job during the initial 12-week posttreatment follow-up period $(N=22)$, the mean number of weeks worked during the 6-month posttreatment follow-up period was $16.4(S D=10.3)$. Of these persons, 3 (13.6\%) experienced a job loss and did not work during the 6-month posttreatment follow-up period. Among the 11 participants who first acquired competitive work during the 6-month posttreatment follow-up period, the mean week of job start was $20.5(S D=8.0)$. Among this group, the mean number of weeks worked during the 6-month posttreatment follow-up period was $12.9(S D=8.5)$.

\section{Steady Work}

Among the 37 participants who worked during the 6-month posttreatment follow-up, 27 participants (73.0\%) were steady workers, as they worked at least $50 \%$ of the follow-up period. Of these, 16 participants (43.2\%) worked continuously across 6 months.

\section{Hours Worked and Wages Earned}

Among the 37 participants who worked during the 6-month follow-up period, mean total weeks worked was 18.68 $(S D=8.62)$ and mean total hours worked was $500.97(S D=334.51)$. Furthermore, as demonstrated in Table 2 and Figures 1 and 2, among the full sample, across the 6-month posttreatment follow-up period, mean hours worked, $F(2,100)=14.89, p=.00$, and mean wages increased, $F(2,98)=22.57, p=.00$. Specifically, participants worked significantly more hours per week in the 6-month posttreatment follow-up period compared with the baseline period (i.e., the12 weeks preceding the study), $t(50)=-5.33, p=.00, d=.78$; and compared with the 12 week, posttreatment follow-up, $t(50)=-2.56, p=.013 ; d=.36$. Similarly, during the 6-month posttreatment follow-up period, mean wages significantly increased, compared with the baseline period, $t(50)=$ $-5.55, p=.00, d=.78$ and the 12 weeks during treatment, $t(49)=-3.0, p=.00, d=.50$. 


\begin{tabular}{|c|c|c|c|c|c|c|}
\hline \multirow[b]{2}{*}{ Competitive work outcomes } & \multicolumn{2}{|c|}{ Baseline } & \multicolumn{2}{|c|}{ 12-week follow-up } & \multicolumn{2}{|c|}{$\begin{array}{l}\text { 6-month } \\
\text { posttreatment } \\
\text { follow-up }\end{array}$} \\
\hline & Mean & $S D$ & Mean & $S D$ & Mean & $S D$ \\
\hline & \multicolumn{2}{|c|}{$N=52$} & \multicolumn{2}{|c|}{$N=52$} & \multicolumn{2}{|c|}{$N=51$} \\
\hline Hours worked per week & 4.42 & 9.7 & 9.32 & 13.7 & 13.98 & 13.9 \\
\hline Hourly wages & 2.03 & 4.5 & 6.23 & 5.8 & 9.39 & 9.1 \\
\hline Work productivity items & \multicolumn{2}{|c|}{$N=8$} & \multicolumn{2}{|c|}{$N=22$} & \multicolumn{2}{|c|}{$N=37$} \\
\hline Frequency of losing concentration at work in last 2 weeks & 20.83 & 17.9 & 11.11 & 12.8 & 20.23 & 21.8 \\
\hline Frequency of repeating a job in last 2 weeks & 16.67 & 28.9 & 4.17 & 9.6 & 17.86 & 29.7 \\
\hline Frequency of working more slowly than usual in last 2 weeks & 22.92 & 19.8 & 13.89 & 15.4 & 16.67 & 21.4 \\
\hline Frequency of feeling fatigued at work in last 2 weeks & 31.25 & 24.1 & 29.17 & 30.0 & 32.14 & 27.5 \\
\hline Frequency of doing nothing at work in last 2 weeks & 6.25 & 11.3 & 2.78 & 8.1 & 5.95 & 13.5 \\
\hline Work productivity total & 19.58 & 12.1 & 12.22 & 9.7 & 18.57 & 15.4 \\
\hline
\end{tabular}

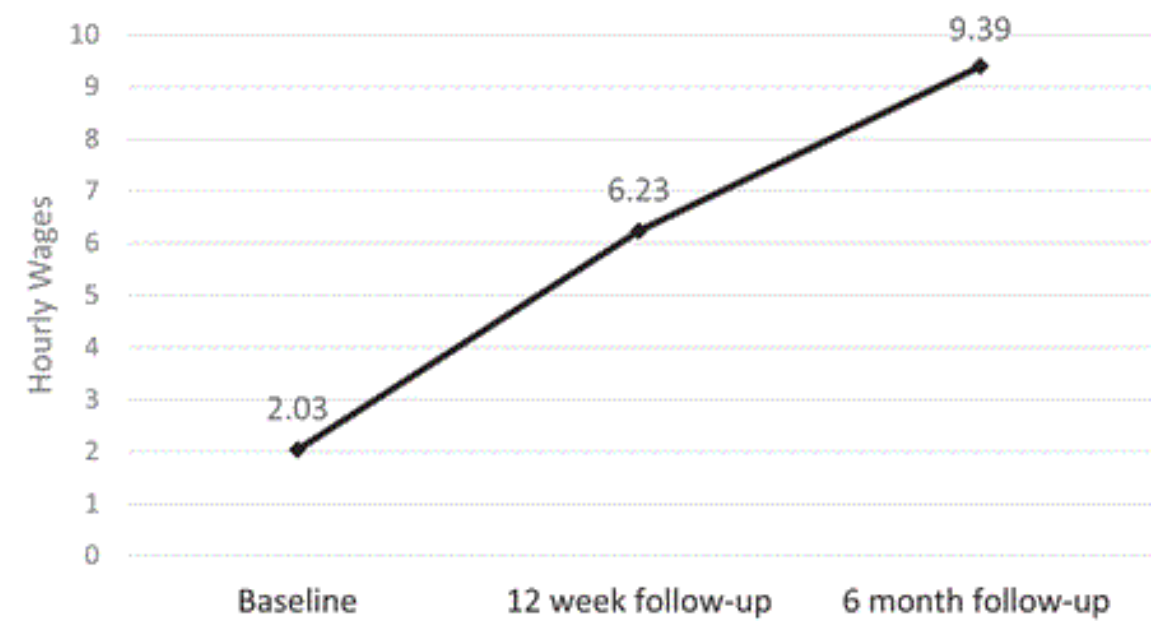

Figure 1. Mean hours worked per week across all study periods, $N=52$.

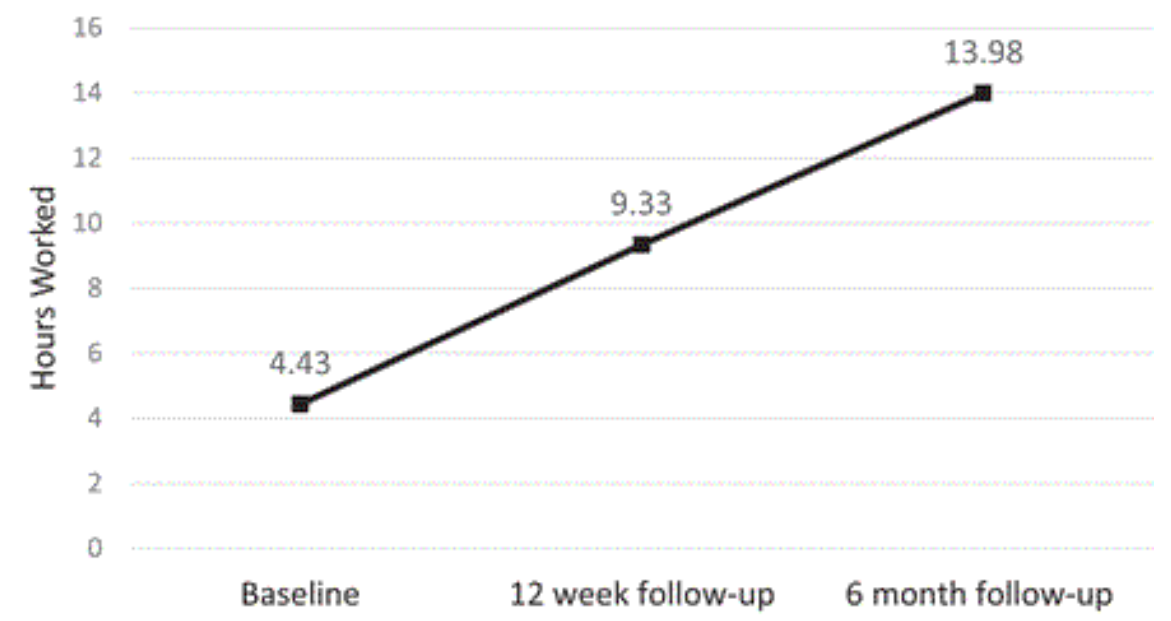

Figure 2. Mean wages earned per hour across all study periods, $N=52$.

\section{Work Effectiveness}

Compared with the 12-week follow-up period in which participants reported over $88 \%$ effectiveness (Kukla et al., 2018), working participants during the 6-month posttreatment follow-up period $(N=37)$ maintained comparably high work effectiveness of $87.6 \%$ (13.71). 


\section{Work Productivity}

As shown in Table 2, participants employed in competitive jobs at baseline reported some disruption in overall work productivity during days at work, $M=19.58(S D=12.14)$. Work productivity improved at 12 weeks, as participants reported lower levels of disruption, $M=12.22(S D=9.74)$. Lastly, work productivity among workers at the 6-month posttreatment follow-up again reflected only slight disruption, $M=18.57(S D=15.42)$.

\section{Discussion}

The current findings add to our developmental pilot work by establishing that CBTw is associated with potential benefits sustained 6 months beyond the intervention period in the areas of competitive job acquisition, duration of work and income earned from work, and attainment of steady employment. Perhaps the most encouraging finding was regarding job acquisition, as three-quarters of unemployed participants secured a competitive job during the full study period. This result should be considered in the context-at baseline, the mean time participants had been receiving supported employment services was 1 year, a substantial period of time without obtaining a job. Further, this competitive job attainment is substantially higher than the one third of veterans receiving supported employment services alone who obtain competitive work at the time of service closure, according to national VA program evaluation data (Abraham et al., 2017). Considered together, these findings may suggest that CBTw is a promising augment to vocational services and worthy of further, more rigorous study.

Also promising was that over $70 \%$ of participants became steady workers during the 6-month posttreatment follow-up period. This is higher than nearly $40 \%$ of veterans with posttraumatic stress disorder receiving VA supported employment services alone who became steady workers across 18 months in a recent randomized controlled trial (Davis et al., 2018). In addition, the significant increases in average hours worked and wages earned suggest more consistent and potentially higher quality work over time. Among the eight workers at baseline, all maintained their jobs at the 6 months posttreatment, and only three participants (out of 22 participants) who initially obtained work during the 12-week intervention period experienced a job loss. That is, after attaining competitive jobs, most participants worked the remainder of the study period, with increasing time and wages; job losses and subsequently falling out of the workforce during the study were infrequent. In total, these results establish a positive work trajectory and compare favorably to typical job tenure periods of a few months (Bond \& Kukla, 2011).

A comparison of on-the-job outcomes at each assessment period demonstrated improved work effectiveness numbers at posttreatment (Kukla et al., 2018) that maintained to the 6-month posttreatment follow-up. In addition, work performance data indicate some performance disruption at baseline that trended toward improvement at posttreatment and at 6 months posttreatment, although the low sample size (i.e., few workers at baseline) prevents hypothesis testing across time. In general, participants reported only mild disruption across the study period. Yet, it should be noted that the areas in which participants reported the most performance impairment included experiencing fatigue on the job, and loss of concentration. These findings are in line with conclusions among veterans with mental illness and vocational providers regarding the importance of managing physical health and cognitive symptoms at work (Kukla et al., 2015, 2016; Kukla, Rattray, \& Salyers, 2015). CBTw may be a promising intervention to address productivity and effectiveness issues given its emphasis and content tailored to address work success and bolster adaptive coping skills, including the generation of personalized work success management plan that can incorporate these elements. However, given the small number of participants working at baseline and the finding that work productivity returned to near baseline levels at the final follow up point, more research is needed to investigate key on-the-job outcomes associated with CBTw and examine whether effects may be sustained beyond the intervention period. 
This study has several limitations. The study did not include a control group of vocational services only; thus, rigorous testing of CBTw is needed to determine causality and draw substantive conclusions. Future research should examine the impact of CBTw on key indicators of work success using a randomized controlled design, controlling for threats to internal validity. In addition, although this study assessed outcomes 6 months beyond the 12-week intervention period, longer time frames are warranted; this is particularly necessary as improvements in functioning may emerge more slowly over longer periods in adults with serious mental illness. Third, the treatment targets or pathways by which CBTw may have exerted influence on employment are unclear and may be varied; future research should examine the targets in this multifaceted intervention. Lastly, fidelity to the Individual Placement and Support (IPS) model of supported employment was not assessed and the incremental benefits of CBTw in the presence of high fidelity services are unknown. In a similar vein, any changes to IPS services in the context of CBTw were unmeasured. Although IPS modification was not part of the CBTw implementation, it is possible that adjustments were made (e.g., vocational staff persons choosing to reinforce healthy thinking about work during follow up). Future work should examine program adherence to the IPS model and other implementation approaches to further enhance work outcomes associated with CBTw added to employment services in the VA and in community settings serving nonveterans.

In conclusion, this study builds upon the growing preliminary evidence that CBTw coupled with individualized vocational services may be beneficial to a range of work outcomes in adults with mental illness. The results of this study along, with previous findings highlighting strong feasibility and acceptability of CBTw by key stakeholders, including people with psychiatric conditions, vocational providers, and program leadership (Kukla et al., 2018), suggests that CBTw potentially holds promise for further implementation and scalability. Future research including more rigorous study designs is necessary to more fully understand the effects of CBTw and its place within vocational service systems.

\section{References}

Abraham, K. M., Yosef, M., Resnick, S. G., \& Zivin, K. (2017). Competitive employment outcomes among veterans in VHA therapeutic and supported employment services programs. Psychiatric Services, 68, 938-946. 10.1176/ appi.ps.201600412

Adler, D. A., Possemato, K., Mavandadi, S., Lerner, D., Chang, H., Klaus, J., . . Oslin, D. W. (2011). Psychiatric status and work performance of veterans of Operations Enduring Freedom and Iraqi Freedom. Psychiatric Services, 62, 39-46. 10.1176/appi.ps.62.1.39

Bell, M. D., Lysaker, P. H., Beam-Goulet, J. L., Milstein, R. M., \& Lindenmayer, J. P. (1994). Five-component model of schizophrenia: Assessing the factorial invariance of the Positive and Negative Syndrome Scale. Psychiatry Research, 52, 295-303.

Beck, A. T., \& Steer, R. A. (1990). Manual for the Beck Anxiety Inventory. San Antonio, TX: Psychological Corporation.

Beck, A. T., Steer, R. A., \& Brown, G. K. (1996). Manual for the Beck Depression Inventory-II. San Antonio, TX: Psychological Corporation.

Bond, G. R., \& Kukla, M. (2011). Is job tenure brief in Individual Placement and Support (IPS) employment programs?Psychiatric Services, 62, 950-953. 10.1176/ps.62.8.pss6208_0950

Burnett-Zeigler, I., Ilgen, M. A., Bohnert, K., Miller, E., Islam, K., \& Zivin, K. (2013). The impact of psychiatric disorders on employment: Results from a national survey (NESARC). Community Mental Health Journal, 49, 303-310. 10.1007/s10597-012-9510-5 
Corbière, M., Lanctôt, N., Sanquirgo, N., \& Lecomte, T. (2009). Evaluation of self-esteem as a worker for people with severe mental disorders. Journal of Vocational Rehabilitation, 30, 87-98.

Davis, L. L., Kyriakides, T. C., Suris, A. M., Ottomanelli, L. A., Mueller, L., Parker, P. E., . . the VA CSP \#589 Veterans Individual Placement and Support Toward Advancing Recovery Investigators. (2018). Effect of evidence-based supported employment vs transitional work on achieving steady work among veterans with posttraumatic stress disorder: A randomized clinical trial. Journal of the American Medical Association Psychiatry, 75, 316-324. 10.1001/jamapsychiatry.2017.4472

Kay, S. R., Fiszbein, A., \& Opler, L. A. (1987). The Positive and Negative Syndrome Scale (PANSS) for schizophrenia. Schizophrenia Bulletin, 13, 261-276.

Kukla, M., Bonfils, K. A., \& Salyers, M. P. (2015). Factors impacting work success in Veterans with mental health disorders: A Veteran-focused mixed methods pilot study. Journal of Vocational Rehabilitation, 43, 51-66. 10.3233/JVR-150754

Kukla, M., McGuire, A. B., \& Salyers, M. P. (2016). Barriers and facilitators related to work success for veterans in supported employment: A nationwide provider survey. Psychiatric Services, 67, 412-417. 10.1176/appi.ps.201500108

Kukla, M., Rattray, N. A., \& Salyers, M. P. (2015). Mixed methods study examining work reintegration experiences from perspectives of Veterans with mental health disorders. Journal of Rehabilitation Research and Development, 52, 477-490. 10.1682/JRRD.2014.11.0289

Kukla, M., Strasburger, A. M., \& Lysaker, P. H. (2016). A CBT intervention targeting competitive work outcomes for persons with mental illness. Psychiatric Services, 67, 697. 10.1176/appi.ps.670504

Kukla, M., Strasburger, A. M., Salyers, M. P., Rattray, N. A., \& Lysaker, P. H. (2017). Subjective experiences of the benefits and key elements of a cognitive behavioral therapy focused on community work outcomes in persons with mental illness. Journal of Nervous and Mental Disease, 205, 66-73. 10.1097/NMD.0000000000000601

Kukla, M., Strasburger, A. M., Salyers, M. P., Rollins, A. L., \& Lysaker, P. H. (2018). A pilot test of group based cognitive behavioral therapy to augment vocational services for persons with serious mental illness: Feasibility and competitive work outcomes. Journal of Nervous and Mental Disease, 206, 310-315. 10.1097/NMD.0000000000000796

Pogoda, T. K., Stolzmann, K. L., Iverson, K. M., Baker, E., Krengel, M., Lew, H. L., . . Meterko, M. (2016). Associations between traumatic brain injury, suspected psychiatric conditions, and unemployment in Operation Enduring Freedom/Operation Iraqi Freedom veterans. The Journal of Head Trauma Rehabilitation, 31, 191-203. 10.1097/HTR.0000000000000092

Stewart, W. F., Ricci, J. A., Chee, E., Hahn, S. R., \& Morganstein, D. (2003). Cost of lost productive work time among U.S. workers with depression. Journal of the American Medical Association, 289, 3135-3144. 10.1001/jama.289.23.3135

Williams, A. E., Fossey, E., Corbière, M., Paluch, T., \& Harvey, C. (2016). Work participation for people with severe mental illnesses: An integrative review of factors impacting job tenure. Australian Occupational Therapy Journal, 63, 65-85. 10.1111/1440-1630.12237 
Zivin, K., Bohnert, A. S., Mezuk, B., Ilgen, M. A., Welsh, D., Ratliff, S., . . Kilbourne, A. M. (2011). Employment status of patients in the VA health system: Implications for mental health services. Psychiatric Services, 62, 3538. 10.1176/ps.62.1.pss6201_0035

Zivin, K., Yosef, M., Levine, D. S., Abraham, K. M., Miller, E. M., Henry, J., . . .Valenstein, M. (2016). Employment status, employment functioning, and barriers to employment among VA primary care patients. Journal of Affective Disorders, 193, 194-202. 10.1016/j.jad.2015.12.054

Submitted: October 15, 2018 Revised: February 20, 2019 Accepted: February 24, 2019 